\title{
CFD-Based Selection of Dispersion Plates
}

\author{
CHEN Jing $^{1, \mathrm{a}}$, DENG Ning ${ }^{2}$, WU Guoyong ${ }^{3}$, ZHOU Jinqing ${ }^{4}$ \\ ${ }^{1}$ LiuZhou Vocational \& Technical College, Liuzhou, Guangxi 545006, China \\ ${ }^{2}$ Liuzhou City Vocational College, Liuzhou, Guangxi 545002, China \\ ${ }^{3}$ Guangxi Science \& Technology Normal University, Laibin, Guangxi 546199, China \\ ${ }^{4}$ Liuzhou HUGEST Chemical Machinery Co., Ltd, Liuzhou, Guangxi 545005, China
}

\begin{abstract}
Computational Fluid Dynamics (CFD) technology was used to analyse dispersion effects of 3 plates of different structures during adhesives production. Three models of the dispersion plates were designed: (1) Project 1: a plate with sharp and short teeth, (2) Project 2: a plate with sharp and long teeth, (3) Project 3: a plate with blunt and long teeth. Pictures of velocity flow contours and flow trajectories in the CFD analysis were obtained to show the flow conditions inside the Reactor. Particle studies were also run to predict adhesive particles dispersion effects of these 3 kinds of plates, so that the best structure of the plate would be chosen.
\end{abstract}

\section{Introduction}

During the preparation of adhesives or battery materials, mixers were needed to mix raw materials evenly. With the development of industry, the amount of polymer melt with high viscosity increases rapidly, so do the requirements for mixing equipment [1]. Dispersion plates were recommended to use in the reactor, so that the raw material could be mixed more evenly,. But how to design and choose more efficient dispersion plates? With the help of CFD (Computational Fluid Dynamics) technology, a better dispersion plate can be easily selected before doing a large number of physical experiments and tests.

In this paper, SolidWorks Flow Simulation was used to analyze three kinds of dispersion plates with different structures: (1) Project 1: a plate with sharp and short teeth, (2) Project 2: a plate with sharp and long teeth, (3) Project 3: a plate with blunt and long teeth. Pictures of velocity flow contours, flow trajectories and particle studies in the CFD analysis were obtained to show the flow conditions inside the reactor. Therefore, the goal to evaluate the dispersion effect of the three different dispersal plates is achieved.

\section{Premise \& Simplification}

\subsection{Simplified model}

Based on previous industrial experience, three dispersed plate CAD models with different structures are designed: (1) Project 1: a plate with sharp and short teeth, (2) Project 2: a plate with sharp and long teeth, (3) Project 3: a plate with blunt and long teeth (Fig. 1 (a) - (c)). In order to predict the mixing effect of these three models, it is necessary to place each plate into the same environment condition. Take the $100 \mathrm{~L}$ reactor for example, the reactor should have two identical dispersion plates with the same structure fastened on the same rotating shaft. In the reactor, stirring slurry, emulsifying head, bolts and other components are omitted to simplify the calculation. The assembly analysis object is shown in Fig. 1 (d).

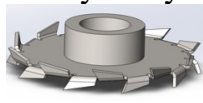

(a) Structure 1

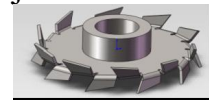

(b) Structure 2

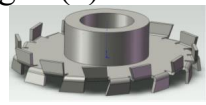

(c) Structure 3

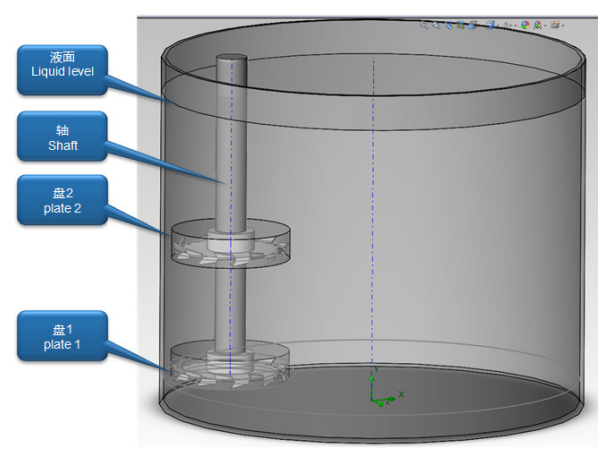

(d) Plates Assembled in the reactor

Figure 1.Below the figure.Structure of 3 dispersion plates and they are inside the reactor

Each a analysis object model consists of a reactor, a shaft and two identical dispersion plates (the plate near the bottom is named plate 1, while the one far away from the bottom is named plate 2). The inner diameter of the reactor is $596 \mathrm{~mm}$, the distance between the plate 1 and the bottom is $44 \mathrm{~mm}$, the distance between the plate 1 and the plate 2 is $177 \mathrm{~mm}$, the distance between the shaft

a Corresponding author: CHEN Jing, 1936307477@qq.com; 
and the center of the reactor is $204 \mathrm{~mm}$, and the height of the liquid from the bottom is $450 \mathrm{~mm}$. To save compute time, only set the half part of the reactor reduce the computational domain size and grid number. In the actual stirring process, the viscosity of the solution is a dynamic value with the effect of temperature and shear, so that a new dynamic viscosity table is needed to build.

\subsection{Premise of simulation}

In the CFD analysis, different viscosities, densities, temperatures and other physical properties of fluids can be individually set. The truth is, with the different rotational speeds of the dispersion plate, the obtained flow conditions in the reactor under different boundary conditions are different too. During the preparation of adhesives or battery materials, the role of the dispersion plates is to disperse the suspension and particles evenly, since the mass flow rate of suspended particles is quite small relative to the whole mass flow, so it has very little effect on the whole flow.

To simplify the project, a standard $\kappa-\varepsilon$ double equation model is chosen since the fluid is only air. Addition assumptions are as follows: (1) physical parameters between solid and fluent are set as constants; (2) fluent is set as turbulence; (3) only compute the half part of the reactor reduce the computational domain size and grid number; (4) ignore the influence of the flow temperature; (5) The tracer particles have no effect on the flow field, there is no interaction between the tracer particles and the movement of the tracer particles is completely determined by the flow field [2-3, 7-12].

\section{Pre-treatment}

\subsection{Definition of Drag coefficient \& Boundary conditions setting}

According to the 'guide 'in Flow Simulation, the initial settings are as follows: the SI length unit is $\mathrm{mm}$, the analysis type is internal flow, and the cavity without flow conditions is excluded. According to the actual flow, the fluid has a dynamic value, which is affected by the temperature and shear. Therefore, a dynamic viscosity was written manually. Boundary Conditions are set as follows: (1)Plate 1 and Plate 2 are set to be the rotating regions, (2)rotating speed is 3000rpm (anti-clockwise from top to bottom), (3)the inner wall and bottom of the barrel are set to be stationary, (4)the liquid surface is set to atmospheric pressure (ambient pressure).

\subsection{Mesh \& Solve}

The model does not need to be totally meshed, only the half part with the dispersed plates should be meshed. Under the global automatic setting, the "initial mesh level" can be set to 5 levels of accuracy. Because the thickness of the dispersion plate is $2 \mathrm{~mm}$, the "minimum wall thickness" can be set to $1 \mathrm{~mm}$, the mesh near the filter plate can be refined, and the "optimized thin-walled surface solution" can be selected. The area around the disk 2 is set up to refine the mesh in order to get a smaller grid of key parts. After meshing, more than 400,000 fluid grids and 170,000 partial grids (i.e. grids with solid and fluid boundaries) were obtained.

\section{Results}

\subsection{Velocity contours (extract the middle section)}

Fig. 2 is the velocity contours around the plate 1 of the three structures. The velocity contours reveal that the highest velocity occurs around the plate tooth. According to the velocity contours of the three structures, in Structure 1, there was a red "higher velocity region" between the plate teeth and the narrow space of the inner wall of the reactor. In Structure 2, the "higher velocity region" was not as obvious as Structure 1 . However, in Structure 3 there was no such "high velocity area" at all.

Figure 3 shows the velocity contours around the plate 2 of the three structures. After comparison, it is clear to find out that the "velocity influence region" of the plate 2 in the Structure 2 is the largest, followed by the Structure 1 , and the "velocity influence region" of the Structure 3 is the smallest.

Fig. 4 is a velocity section of the front view of the three structures. After comparison, it is clear to find out that both the "higher velocity region" and "velocity influence region" of Structure 2 are the largest, followed by structure 1, then Structure 3 .

Such results quite possibly related to the structure of the teeth: Structure 1 is oblique, structure 2 is equivalent to increasing the height of the tooth surface on the basis of structure 1, structure 3 is high, but the inclination is small (Fig. 1 (a) - (c)). Structural 3 has no sharp teeth shape, so in the same rotational velocity and fluid, the influence of structural 3 on the surrounding fluid is the smallest, which is also very consistent with the actual situation.
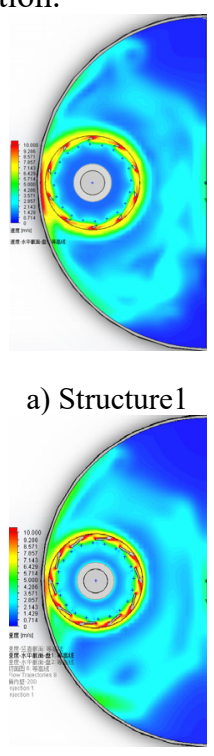

c) Structure2

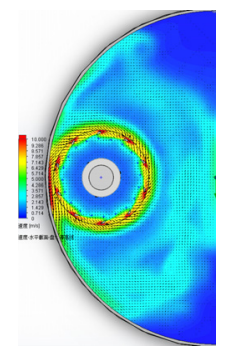

b)Structure 1(with vector)

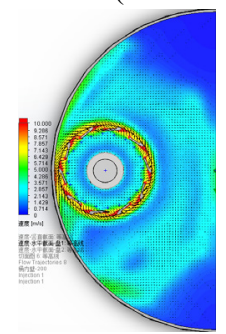

d)Structure 2 (with vector) 


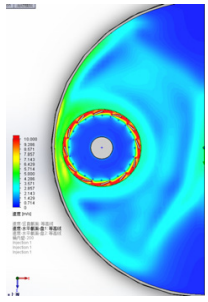

e) Structure 3

Figure 2 Velocity contours of plate 1 of the three structures

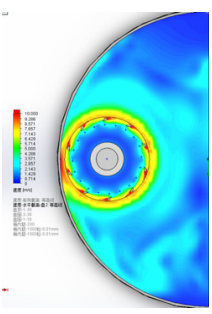

a)Structure1

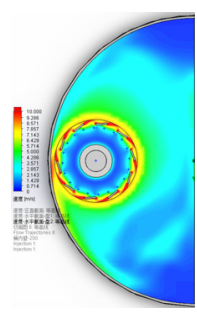

c)Structure2

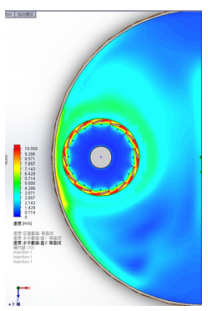

e)Structure 3

Figure 3 Velocity contours of plate 2 of the three structures

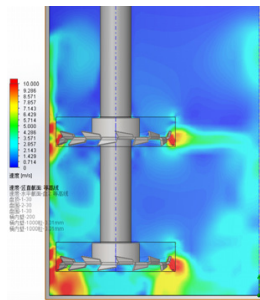

a) Structure1

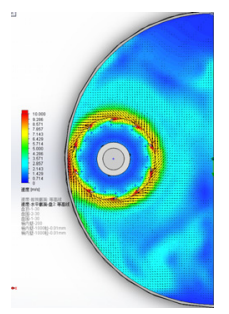

b)Structure1 (with vector)

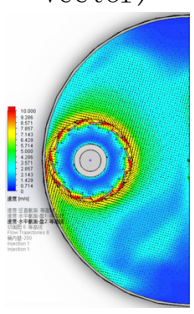

d)Structure2 (with

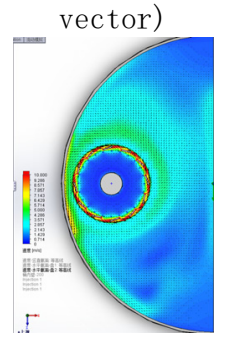

Structure 3 (with vector)

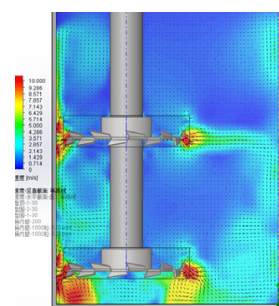

b)Structure1(with vector)

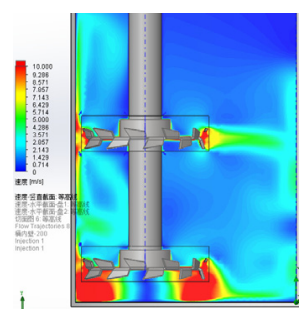

c)Structure 2

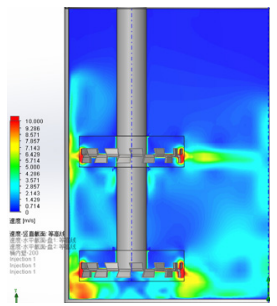

e)Structure 3

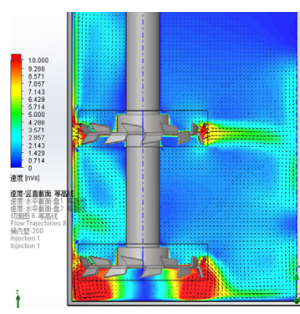

d)Structure 2(with

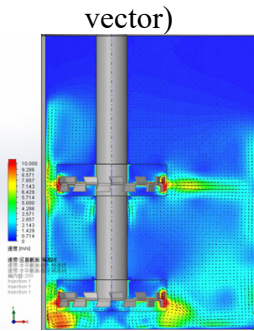

f)Structure 3(with vector)
Figure 4 Velocity contours of FRONT of the three structures

\subsection{Flow trajectories and particle tracer study}

In Fig.5, there were two kinds of pictures, and the left column visualizes the distribution of the velocity contours with arrows, while the right column shows the particle tracer study. The color in the diagram indicates the velocity of the fluid, while the arrow reveals the direction of the fluid. Flow Simulation can simulate the flow in the reactor more vividly in animation. In Fig.5 a) c) e), the red region means the highest velocity of the flow. It can be seen from the picture that the red fastest regions are always around the dispersion plates. A vortex was formed beside each dispersion plate because of shaft rotation near the wall. Comparing the three structures, under the same conditions, flow in Structure 2 was the fiercest followed by Structure 1 and then Structure 3, which is consistent with the conclusion of velocity distribution contours.

In Fig. 5 b) d) f) show the "particle studies" which can predict the dispersion effect of suspension and material particles. The "particle studies" can obviously simulate the movement condition in the reactor [4]. In the three projects, setting are almost the same: (1) 200 points, (2) diameter of particles is $0.01 \mathrm{~mm}$, (3)particles display diameter is $3 \mathrm{~mm}$. After calculation, we can see that in all the three projects, due to the gravity, there are more particles gathering around dispersion plate 2 than plate 1 .

After comparison, it can be observed that: (1) the particle velocity around the dispersion plate 1 in Structure 2 is the fastest, followed by Structure 1 and then Structure 3; (2) the particle velocity around the dispersion plate 2 in Structure 2 is the fastest, followed by Structure 1 and then Structure 3; (3) due to the gravity, particles around plate 2 are more than plate 1 . As for the particles of slow velocity, they may be quite possibly become deposits. 


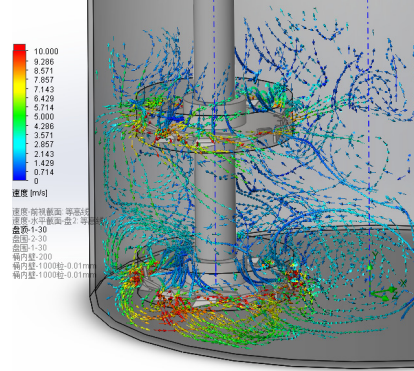
trajectories

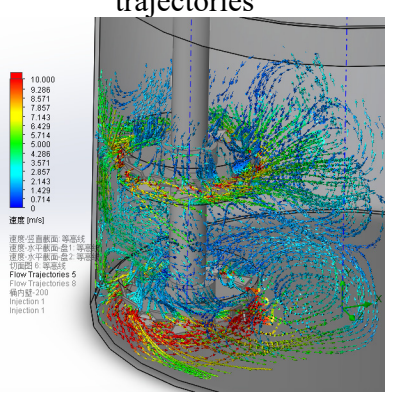

c)Structure 2: flow trajectories

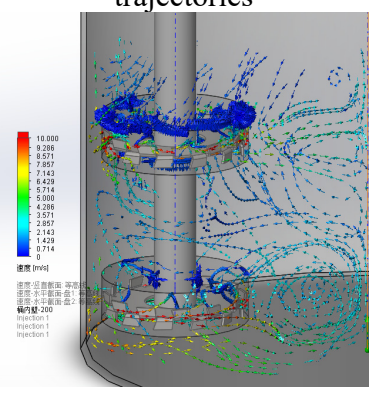

e)Structure 3: flow trajectories
a)Structure 1: flow

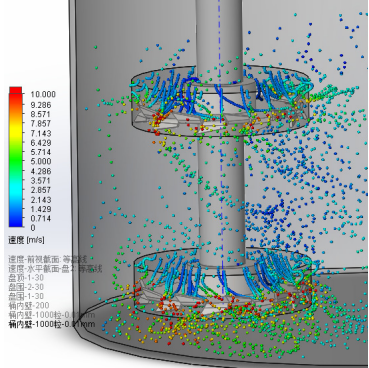

b)Structure 1: particle results

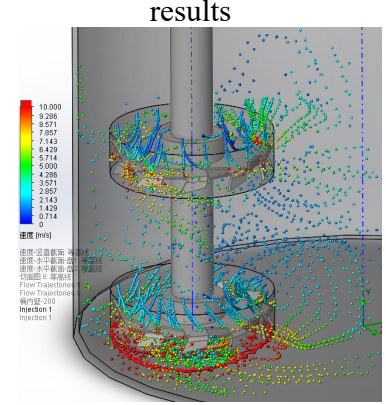

d)Structure 2: particle results

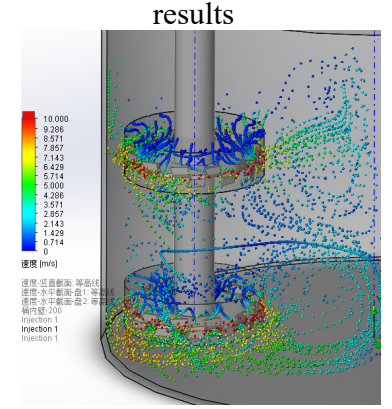
results
f)Structure 3: particle

Figure.5 Flow trajectories inside

\section{Conclusions}

SolidWorks Flow Simulation can well simulate complex fluids in reactors. Through the post-treatment, conditions are as follows:

(1) In Figure. 3 the "velocity influence region" of the plate 2 in the Structure 2 is the largest, followed by the Structure 1, and the "velocity influence region" of the Structure 3 is the smallest.

(2) In Figure. 4 is a velocity section of the front view of the three structures. After comparison, it is clear to find out that both the "higher velocity region" and "velocity influence region" of Structure 2 are the largest, followed by structure 1, then Structure 3.

(3) In Figure.5, both the "flow trajectories" and "particle tracer study" pictures have indicated that velocity of flow and particles around the dispersion plate 1 in Structure 2 is the fastest, followed by Structure 1 and then Structure 3.

(4) Due to the gravity, particles around plate 2 are more than plate 1 . As for the particles of slow velocity, they may be quite possibly become deposits.
The CFD technology can intuitively predict the mix effect of the dispersing plate. In the actual preparation process of the adhesive, the best dispersing plate is selected and provides a powerful reference for optimizing the product structure design.

\section{Acknowledgment}

1. Enhance Project of Middle-aged and Young College Teachers of Guangxi, China (2018KY0990); 2. The software of SolidWorks provided by New Well Technology Co. Ltd.

\section{References}

1. Jing Chen,(2017) 'CFD-based optimization of truck fairing structure',IOP Conference Series: Earth and Environmental Science,Volume 61, conference 1 .

2. Li Cuiping,(2014) 'Optimization design of FSAE racer body based on CFD', Journal of Machine Design, Vol. 31, No.8, pp74-77.

3. Wang Jun, (2013) 'Application of CFD analysis method in Vehicle design'. Automobile Technology, No.4, pp 17-20.

4. Jian zhong Xiao-Ming-Cai-*-William-James-Bloss. (2016) 'Coupling dynamics and chemistry in the air pollution modelling of street canyons: A review'. Environmental Pollution, Vol.214.

5. M. lateba R.N.-Meroneyb-M. -Yataghenec-H.Fellouahd-F.-Saleha- M.C.-Boufadela. (2016) 'On the use of numerical modelling for near-field pollutant dispersion in urban environments ? A review'. Environmental Pollution, Vol. 208, pp 271283.

6. Jorge d. cambaa Manuel-Conterob-Pedro-Companyc. (2016) 'Parametric CAD modeling: An analysis of strategies for design reusability'. Computer-Aided Design, Vol.74, pp18-31.

7. Hamid nawaz Yan-Sheng-Yuan. (2013) 'Thermal Comfort Analysis of a Ship Air-Conditioning System Using Solidworks Flow Simulation'. Advanced Materials Research, Vol.773, pp 883-888.

8. Lee T,Filipi Z.( 2011) 'Synthesis of Real-world Driving Cycles Using Stochastic Process and Statistical Methodology'. International Journal of Vehicle Design, Vol.57, No.1, pp 17-36.

9. Paramadayalan T,Pant A. (2015) 'Transient Cfd Model of Full Twc Converter'. International Journal of Vehicle Design, Vol.68, No.4.

10. Saleh Saad-N.1,Barghi Shahzad2(sbarghi2@eng.uwo.ca).(2016) 'Reduction of fine particle emission from a prilling tower using CFD simulation'. Chemical Engineering Research and Design, 109: 171-179.

11. Paramadayalan Thiyagarajan,Pant Atul. (2016) CFD model of multi-converter HC adsorber-TWC system'. International Journal of Vehicle Design, Vol.70, No.4, pp 358-376. 
12. Chen Lei1,Chen Youhua,Huang Kail. (2016) 'Investigation of effective thermal conductivity for pebble beds by one-way coupled CFD-DEM method for CFETR WCCB'. Fusion Engineering and Design, Vol.106, pp 1-8.

13. Danca Calogero,Mancuso Antonio,Mariotti Gabriele-Virzi. (2005) 'Optimisation of a vehicle shape by CFD code'. International Journal of Vehicle Design, Vol.38, No.1, pp 26-41.

14. Chen Jing,(2015) 'Analysis of CFD automobile Three-way catalytic converter'. Environmental Engineering, (12, pp 90-94.

15. Chen Jing. (2016) 'Feeding ball valve optimized by CFD technology. China Adhesives, No.2, pp 16-20.

16. Kiani M,Shiozaki H,Motoyama K. (2015) 'Simulation-based Design Optimisation to Develop a Lightweight Body-in-white Structure Focusing on Dynamic and Static Stiffness'. International Journal of Vehicle Design, Vol.67 No.3.

17. Jing Chen. 'An analysis method of vehicle shape optimization based on CFD technology' China, 201510239905.9 Patent.

18. Chen Jing. (2013) 'Dispersion plate selected by Flow Simulaiton plugin from SolidWorks software'. China Adhesives, No.12, pp 13-16.

19. Matthew chua Chee-Kong-Chui-Constance-Teo. (2015) Computer aided design and experiment of a novel patient-specific carbon nanocomposite voice prosthesis'. Computer-Aided Design, Vol.59, pp 109-118.

20. Min-feng sung Chin-Fu-Chen-Chao-Jung-Chen. (2015) 'Using the CFD Technique to Analyze Tire Tread Hydroplaning Effects'. Asian Journal of Engineering and Technology, Vol.3, No.3, pp 151157.

21. Yan luximon Roger-M.-Ball-\&-Eric-H.C.-Chow. (2015) A design and evaluation tool using 3D head templates'. Computer-Aided Design and Applications, Vol. 13, No.2, pp 153-161. 